\section{Oh to be twenty seven again}

Peter Parham

Discrimination by $\mathrm{T}$ cells of friend and foe is based on displays of peptides derived from intracellular and extracellular proteins. By its generality this mechanism gets antigenpresenting molecules of the immune system involved with almost everything else in the cell. In turn, the pursuit of antigen processing and presentation forces immunologists to sail from the coastal comforts of the plasma membrane and explore the cell's interior.

This coming about was reflected at a workshop last month*, where interest centred on the natural peptides bound by antigenpresenting molecules, the molecular mechanisms of peptide binding and the cellular compartments in which it takes place. In this setting $T$ cells were a generic readout of function, the thymus was rarely mentioned and cellular interactions were, well, almost infra dig (D. Y. Loh, Washington University). Knowledge of the primary and three-dimensional structures of bound peptides in vivo is rapidly advancing, and a newly discovered protein, involved in the assembly of class $I$ antigen-presenting molecules, is firmly on the scene.

Early running in this area was set by studies on the T-cell response to exogenously supplied antigens. Such antigens are mainly presented by class II major histocompatibility complex (MHC) molecules and it was for class II that the dependence of T-cell recognition on binding of degraded antigen fragments to MHC molecules was established. This principle was subsequently shown to extend to class I molecules, but with nuances in mechanism that favour the presentation of intracellular antigens. One difference is that class I molecules bind peptides near their site of synthesis in the endoplasmic reticulum (ER), whereas class II molecules are targeted to compartments of the endocytic pathway by the invariant chain. This trimeric molecule binds class II molecules in the ER, takes them to endosomal and lysosomal compartments for peptide binding, and is itself destroyed in the process (P. Cresswell, Duke University).

A search has been made for possible chaperonin or targeting molecules that interact with class I molecules during their biogenesis. From crosslinking experiments a good case can now be made for transient association of class I heavy chains with a polypeptide of relative molecular mass 88,000 (p88) (D. B. Williams, University of Toronto). In the ER, each newly synthesized class I heavy chain rapidly forms a 1:1 complex with p88. This association does not require prior association with $\beta_{2}$-microglobulin (the second and invariant polypeptide of the class I molecule) and is not perturbed by its subsequent

* Molecular and Cellular Biology of Antigen Processing and Presentation, Cancer Research Institute, New York, 6-7 May, 1991. binding. The signal that triggers release of class I molecules from the $\mathrm{p} 88$ complex is not yet known, but dissociation correlates precisely with the acquisition of terminal sugars in the medial Golgi. These kinetics, combined with experiments implicating the class I transmembrane region in $\mathrm{p} 88$ interactions, imply that $\mathrm{p} 88$ does not directly facilitate folding of class I molecules but acts to retain them in the ER, perhaps until they are loaded with peptide. Another possibility, based on similarities in molecular weight and protease sensitivity, is that $\mathrm{p} 88$ is the product of one of the putative peptide transporter genes encoded by the MHC (see my News and Views article in Nature 351, 271-272; 1991). Intimate association between peptide pump and nascent class I molecules could pinpoint delivery of peptides and in this manner facilitate the correct assembly of class I molecules.

Determination of the three-dimensional structure of a third human class I antigenpresenting molecule, HLA-B27, is nearing completion (D. C. Wiley, Harvard University). Although the product of a different genetic locus, HLA-B27 has a peptide backbone that is essentially the same as that shared by HLA-A2 and HLA-Aw68. This augurs well for a structural generality, because by physicochemical criteria $\mathrm{A} 2$ and B27 are at opposite ends of the spectrum of HLA-A,B diversity. The special contribution of this third class I structure is improved resolution in the 'extra density' of the antigen-binding groove and proof it is a peptide. Whether it be due to the peculiarities of B27, better crystals or third-time luck, this eagerly awaited image of an endogenous peptide is no disappointment. The peptide appears as an extended chain of nine amino acids, not the $\alpha$ helix of popular prediction, with its ends tethered at opposite ends of the groove. Side chains of some amino acids extend deep into specificity pockets formed by variable positions of the class I heavy chain, others point up with the possibility for direct contact with the T-cell receptor. Such contacts, however, remain a threedimensional mystery, as illustrated by systematic analysis of the T-cell receptors interacting with mutant class I molecules and a malarial peptide (P. Kourilsky, Institut Pasteur).

Until last year, almost all studies of the epitopes presented by MHC molecules to T cells used synthetic peptides. From the results for both class I and class II molecules it seemed that optimal epitopes were about 12-15 amino acids in length. Now, characterization of the 'natural' peptides bound by class I molecules within cells shows they are both smaller and more precisely defined than expected (G. van Bleek, Albert Einstein College of Medicine; H.-G. Rammensee,
Max-Planck-Institut für Biologie, Tübingen). Nine amino acids is the current favourite and this number, which is consistent with the crystallography, echoed throughout the workshop. From sequencing both mixtures and individual peptides bound by particular MHC molecules, conserved motifs of 'anchoring residues' - one at the C terminus and another close to the $\mathrm{N}$ terminus - that distinguish sets of binding peptides for different class I molecules are being identified. None of these peptides seems to be longer than nine amino acids. This convergence of crystallography and biochemistry sets a new paradigm for class I epitopes. A key question is whether the same will be true for class II molecules, as there are many suggestions that their binding sites are more accessible to experimentally offered peptide, more tolerant of peptide length and able to undergo reversible peptide exchange as they recycle through the cell (L. Adorini, Sandoz Pharma, Basel; M. M. Davis, Stanford University; B. Pernis, Columbia University).

The unexpectedly short length of naturally presented peptides inevitably raises questions about the biological activities of longer synthetic peptides. Indeed, these 'defined reagents' are not always as advertised. For example, an epitope of Sendai virus was defined using a panel of synthetic 'overlapping' 12-residue peptides derived from the nucleoprotein sequence. Peptides from a single region of the protein were presented to $T$ cells, thereby mapping the epitope. But the active principle in peptide preparations that actually bound to the class I molecules was a very minor constituent. This contaminant was nine amino acids in length, differing from the expected sequence by the absence of the three $\mathrm{N}$-terminal amino acids - the last ones to be incorporated in the synthesis (C. J. Melief and H. L. Ploegh, Netherlands Cancer Institute).

In reevaluation of assays of peptide binding and presentation, smaller peptides are often better. For peptide-driven association of class I molecules in cell lysates (A. Townsend, University of Oxford), for inhibition of peptide binding to the class I molecules of viable cells (B. Barber, University of Toronto) and for reconstitution of HLA-A2 (D. Wiley) nine-residue peptides have more potent activities than their longer, previously advocated, homologues. Clearly, synthetic peptide preparations will now have to be scrutinized closely and, though the immediate prospect is tedious, there are benefits to this turn of events. Synthetic peptides are expensive, and, like yachts, the longer they are the more costly they become. When appropriate endogenous epitopes have been identified, the necessary peptides will not only be cheaper to make, but greatly increased in biological activity and ultimately longer lasting.

Peter Parham is in the Departments of Cell Biology, and Microbiology and Immunology, Stanford University School of Medicine, Stanford, California 94305, USA. 